\title{
Voltage Balancing Control in Three Level Diode Clamped Inverter Using Carrier Based Offset Addition
}

\author{
Michael Raj.P1, Dr.K.Manimala²
}

\begin{abstract}
M.E Scholar: Dept. of Electrical and Electronics Engg., Dr.Sivanthi Aditanar College of Engg.,Tiruchendur, India ${ }^{1}$
\end{abstract}
Professor, Dept. of Electrical and Electronics Engg., Dr.Sivanthi Aditanar College of Engineering.,Tiruchendur, India ${ }^{2}$

\begin{abstract}
Three-level Neutral-Point Clamped (NPC) inverter has become established to be a preferred topology for medium-power motor drive applications operating at several kilovolts. Still several research continues to find solutions to the problem of maintaining a stable neutral-point voltage in the converter. This paper presents the analysis and simulation of a three-level diode-clamped multilevel inverter, employing a sine-triangle modulator with addition of continuous variable offset voltage which regulates the midpoint potential of the dc bus. By maintaining dc-bus voltage balance, a significant reduction in the voltage distortion at the neutral point and also allowing a definitive reduction in the required $\mathrm{dc}$ bus capacitance. The effectiveness of the Pulse Width Modulation (PWM) strategies developed with continuous offset addition is demonstrated by MATLAB/SIMULINK based simulation presented in this paper.
\end{abstract}

Keywords: NPC inverter; 3 phase induction motor; offset addition; carrier based PWM; dq theory; pi control

\section{INTRODUCTION}

Diode clamped/Neutral point clamped multilevel inverter technology has emerged recently as a very important alternative in the area of high-power medium voltage energy control. The structure of diode clamped inverters allows them to reach high voltages and therefore lower voltage rating devices can be used. As the number of levels increases the synthesized output waveform has more steps producing a very fine stair case wave and approaching very closely to the desired sinusoidal wave.

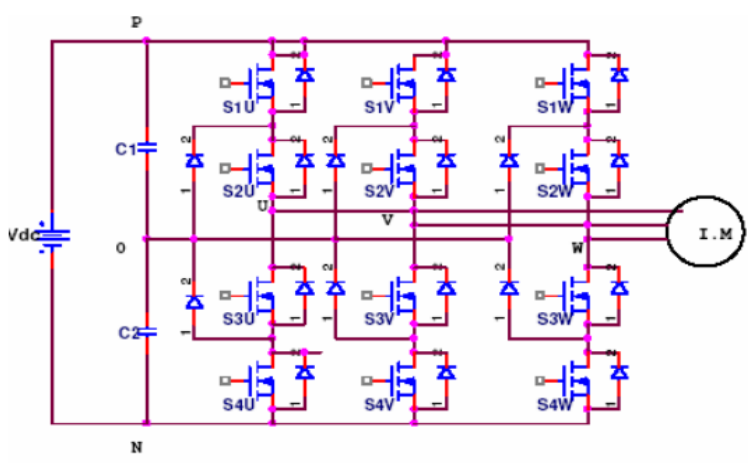

Fig. 1. Three nhase three-level NPC

Hence diode clamped multilevel inverters offer a better choice at a high power end because the high volt-ampere ratings are possible with these inverters without the problems of high $\mathrm{dv} / \mathrm{dt}$ and the other associated ones.

NPC inverter has an inherent problem of unbalanced voltages across dc-link capacitors due to load unbalancing, non uniform distribution of charges, and non-identical properties of dc-link capacitors provided from the manufacturer [1], [2]. Several open loop strategies have been proposed for the reduction of the harmonic content [3], [4].
The authors have proposed two ways of mid-point voltage balancing in NPC three level inverters. One way of voltage balancing based upon the addition or modification of hardware circuitry to the inverter [5]-[10] which modify the charging and discharging currents of DC-link capacitors.

Second way of voltage balancing is based upon modification in inverter control strategy based on PWM schemes. Many carrier and SVPWM based strategies have been proposed for the modulation of theses inverters [11][15].

Table 1: Switching states of three level NPC inverter

\begin{tabular}{|c|c|c|c|c|c|}
\hline S1U & S2U & S3U & S4U & $\begin{array}{l}\text { Switching } \\
\text { states }\end{array}$ & $\begin{array}{l}\text { Voltage } \\
\text { output(Vuo) }\end{array}$ \\
\hline 1 & 1 & 0 & 0 & + & + Vdc/2 \\
\hline 0 & 1 & 1 & 0 & 0 & 0 \\
\hline 0 & 0 & 1 & 1 & - & $-V d c / 2$ \\
\hline
\end{tabular}

A closed loop control strategy, which reduces the harmonic content as well as maintains voltage stability in the neutral point is presented in this paper. Closed loop regulator is based on injecting offset magnitude to the modulating signal as a function of a control input that corrects any existing imbalance.

In this paper main considerations given to regulate the voltage imbalance of NPC inverter employing sinetriangle modulator in conjunction with a closed-loop controller, which considerably reduces the harmonic distortions in the output voltage waveform, resulting in reduction of the required dc bus capacitance. 


\section{NEED OF NEUTRAL POINT BALANCING}

The Neutral point clamped inverter is showed in Fig.1 and Table I give the switching states to generate the three level output voltage for phase U.

Due to unequal voltages across two dc-link capacitors PWM inverter output voltage and output current waveforms get distorted. Unbalance DC link creates increased voltage stress on switching devices. Increased voltage imbalance across dc-link capacitors may cause failure of devices. Therefore, Neutral point voltage balancing control is necessary without sacrificing the harmonic performance of the inverter. Fig. 2(a) and (b) shows the waveforms and harmonic spectrums of phase voltage and

line voltage under burst condition of large imbalance at dc link

with $V \mathrm{dc} 1=200 \mathrm{~V}$ and $V \mathrm{dc} 2=400 \mathrm{~V}$. Under this condition, the dc component and even-order harmonics are more significant which are dangerous for drive and other applications.

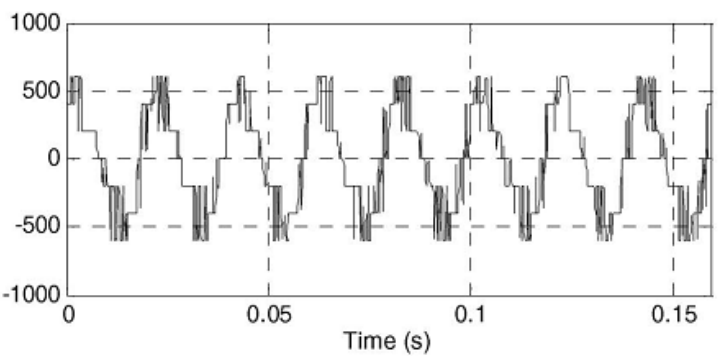

Fig. 2 Output line voltage distortions due to unbalanced DC-link voltage

Table II gives the effect of dc-link imbalance on \% THD with a total dc-link voltage of $400 \mathrm{~V}$. For this study, the dc link was intentionally made unbalanced. It is clear that the line voltage THD will have the minimum value for balanced dc link and they are increasing with increased unbalancing

Table II: Effect of DC link imbalance on output \% THD $(\mathrm{Vdc}=600 \mathrm{~V})$.

\begin{tabular}{|c|c|c|c|}
\hline Vdc1 & Vdc2 & $\begin{array}{c}\text { Fundamental } \\
\text { Line voltage }\end{array}$ & $\begin{array}{c}\text { \%THD line } \\
\text { voltage }\end{array}$ \\
\hline 230 & 370 & 469 & 25.4 \\
\hline 240 & 360 & 471 & 21 \\
\hline 250 & 350 & 492 & 19.7 \\
\hline 300 & 300 & 494 & 16 \\
\hline 350 & 250 & 485 & 16.5 \\
\hline 400 & 200 & 489 & 25 \\
\hline 450 & 150 & 495 & 32.7 \\
\hline
\end{tabular}

\section{DESIGN OF CLOSED LOOP NPC INVERTER}

Designing of closed loop NPC inverter consider the following important aspects,

i) ensuring dc-link capacitor, voltage balancing and regulating dc-link voltage

ii) minimization of inverter voltage and current harmonics; iii) ensuring less and uniform switching stress on switching devices, resulting in reduced switching losses.

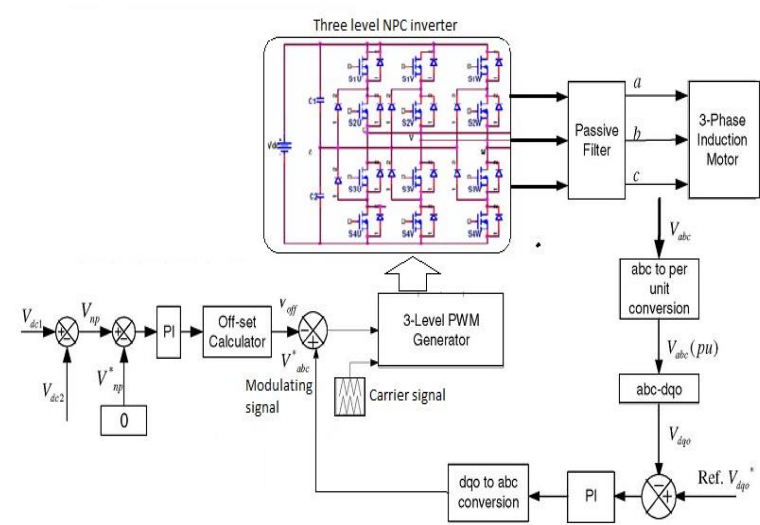

Fig.3 NPC offset addition PWM block diagram

Fig. 3 shows the complete block diagram of the NPC offset addition PWM. It consists of both a dc-link voltage control loop and a load voltage control loop. Three phase load voltages are sensed and converted into a per-unit system. These per-unit voltages are converted into $d q o-$ axis. After comparing with preset values $(\mathrm{Vd}=1, \mathrm{Vq}=0)$ again converted to abc-signal. This Vabc signal is added with the dc-link voltage control loop generated offset signal. This signal is acting as modulating signal. It is compared with carrier PWM by using level shift PWM and the pulses are given to appropriate switches.

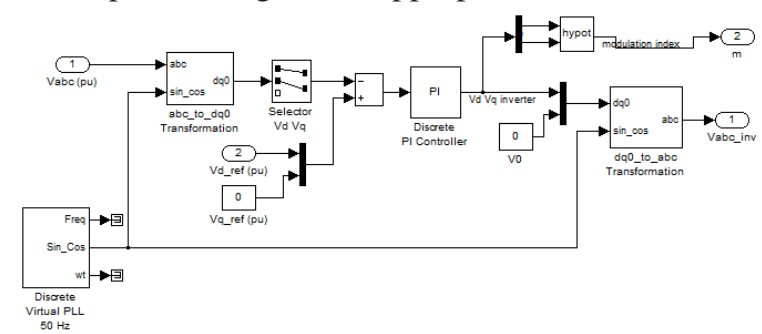

Fig.4 abc to dqo conversion reference comparison and dqo to abc conversion

Fig shows the measured voltage Vabc is converted by using the abc to dqo converstion method using the following three-phase to two-phase conversion:

$V d=2 / 3\left[V a \sin (\omega t)+V b \sin \left(\omega t-120^{\circ}\right)+V c \sin (\omega t-\right.$ $\left.\left.240^{\circ}\right)\right]$

$V q=2 / 3\left[V a \cos (\omega t)+V b \cos \left(\omega t-120^{\circ}\right)+V c \cos (\omega t-\right.$ $\left.\left.240^{\circ}\right)\right]$

$V o=V a+V b+\mathrm{Vc}$

These $d q o$ voltages $V d q o$ are compared with preset values of $d q o$ voltages. It results in voltage error which is processed through a PI controller to generate two axis command signals $V^{*} d q o$. Then, three-phase reference voltage signals are synthesized using the following twophase to three-phase conversion: $\left.V_{a}=V_{d} \sin (\omega t)+V_{q} \cos (\omega t)+V_{o}\right)$ $\left.V_{b}=V_{d} \sin (\omega t-1200)+V_{q} \cos (\omega t-1200)+V_{o}\right)$ $\left.V_{c}=V_{d} \sin (\omega t-2400)+V_{q} \cos (\omega t-2400)+V_{o}\right)$ These are the reference sinusoidal modulating signal $V_{a b c}{ }^{*}$. The amplitude modulation index $m$ is defined as $m=\sqrt{ } V_{d}^{2}+V_{q}^{2}$ 


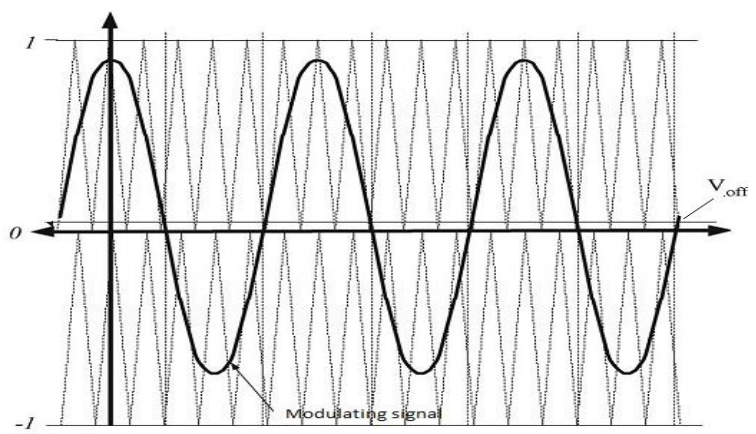

Fig.5 Offset addition PWM

offset addition method is shown in Fig. to the reference sinusoidal signal. The compared signal is given to switches as sequence in the corresponding legs.

\section{SIMULATION RESULTS}

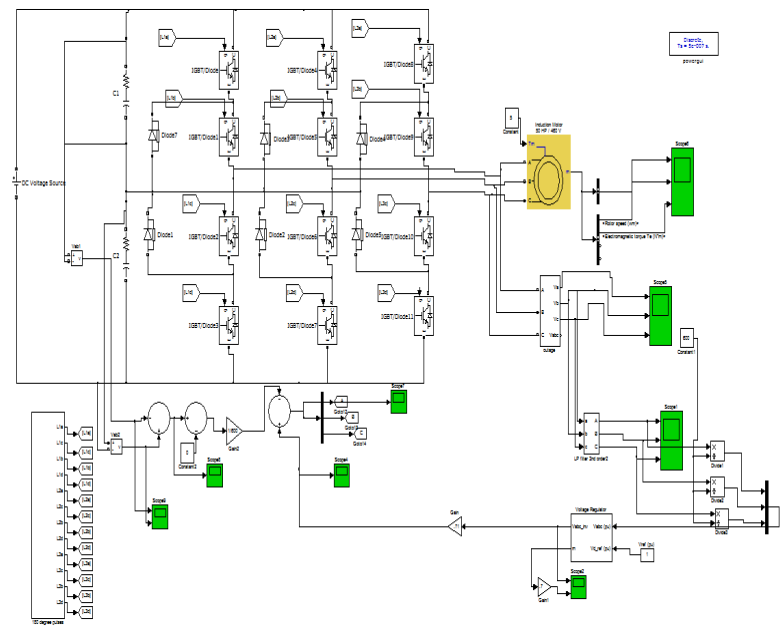

Fig.6 A MATLAB/Simulink model of three-phase three-level DCMLI with the carrier-based offset addition PWM

MATLAB/Simulink model of three-phase three-level DCMLI

with the carrier-based offset addition PWM is shown in fig.6

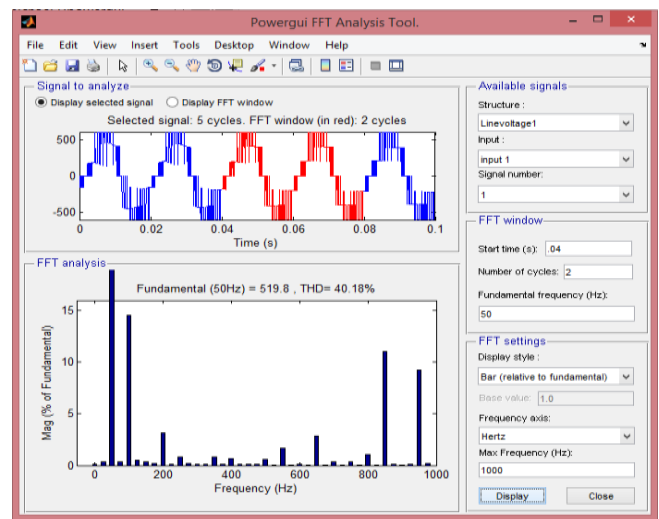

Fig.7 Simulated inverter line voltage (Vab) and its frequency spectrum with unbalanced dc link without closed loop offset PWM and

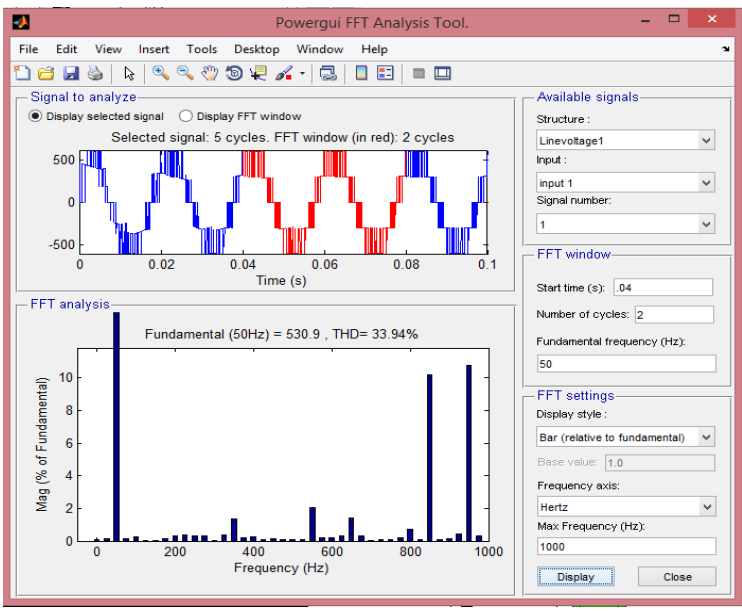

Fig.8 Simulated inverter line voltage (Vab) and its frequency spectrum with unbalanced dc link with Neutral point balancing.

Frequency spectrum of output line voltage without closed loop offset PWM are shown in Fig. 7 with fundamental value as 519.8 and $40.18 \%$ of THD. In Fig.8 frequency spectrum for closed loop NPC inverter with offset addition PWM is shown with fundamenatal value as $530.9 \mathrm{~V}$ and THD values as $33.94 \%$

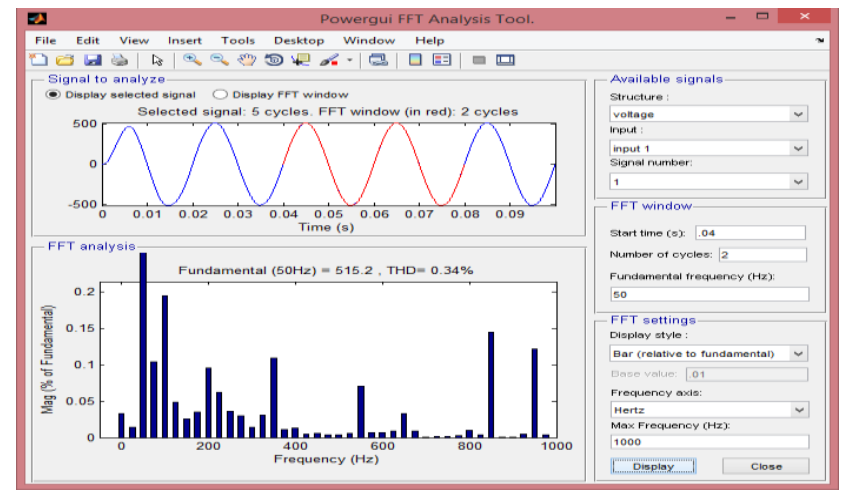

Fig.9 Simulated inverter line voltage (Vab) and its frequency spectrum with unbalanced dc link with neutral point balancing after filtering.

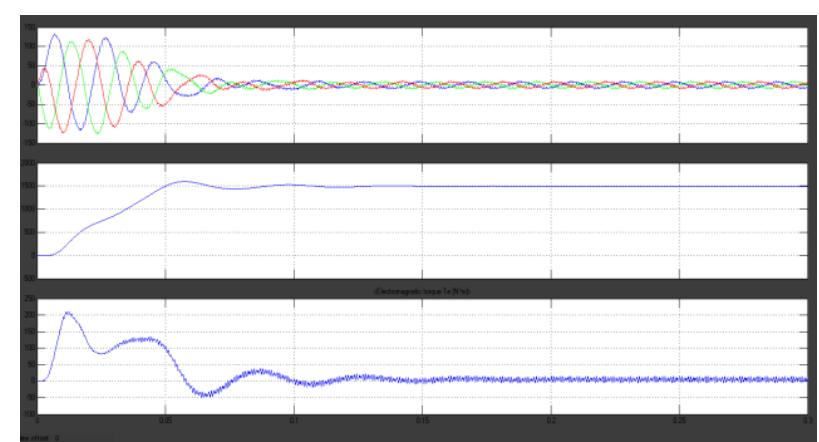

Fig.10 Stator current waveform (Ia, Ib and Ic), Motor speed (rpm), Electromagnetic torque waveforms under balanced dc link voltages after neutral point voltge balancing. 


\section{Vol. 3, Issue 3, March 2015}

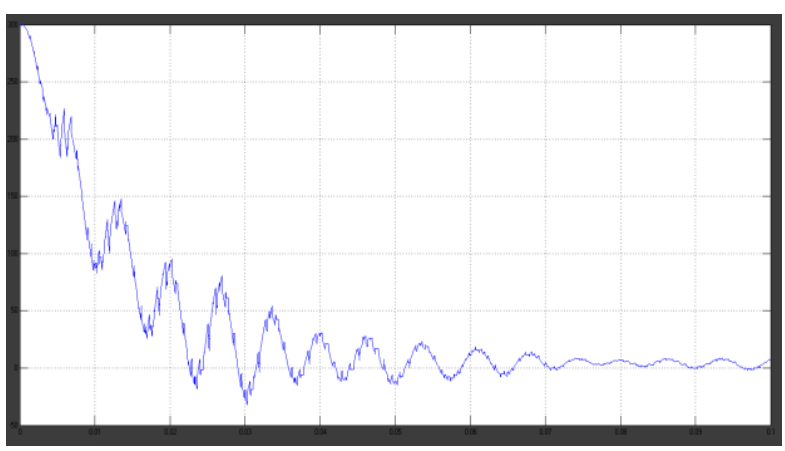

Fig.11 Neutral point voltage (Vnp= Vdc1-Vdc2)

\section{CONCLUSION}

A simple carrier-based neutral voltage balancing for a three-level diodeclamped inverter in conjunction with a closed-loop controller has been proposed in this paper. The proposed offset calculation PWM gives improved inverter performance in terms of reduced THD,with unbalanced dc-link voltages,improved NPP harmonic profile, and balanced dc link with almost zero average Neutral point potential. The novelty of the proposed mehtod is in the determination of the magnitude of variable offset voltagebasedupon the average value, peak-to-peak amplitude,THDs,and third harmonic content in NPP.This not only regulates the NPP but also reduces the harmonic contents in inverter output voltages and currents. Simultaneously, second-order harmonics in inverter output voltage get eliminated which may otherwise produce torque pulsations, harmonic currents, and additional power losses. Aside from maintaining the dc-bus voltage balance, the proposed closed loop offeset voltage PWM leads to a significant reduction in the voltage distortion.

\section{REFERENCES}

[1] J. R. Rodríguez, S. Bernet, B. Wu, J. O. Pontt, and S. Kouro, "Multilevel voltage source converter topologies for industrial medium voltage drives," IEEE Trans. Ind. Electron., vol. 54, no. 6, pp. 2830-2945, Dec. 2007.

[2] J. R. Rodríguez, J.-S.-S. Lai, and F. Z. Peng, "Multilevel inverters: A survey of topologies, controls, and applications," IEEE Trans. Ind.Electron., vol. 49, no. 4, pp. 724-738, Aug. 2002.G. Eason, B. Noble, and I.N. Sneddon, "On certain integrals of Lipschitz-Hankel type involving products of Bessel functions," Phil. Trans. Roy. Soc. London, vol. A247, pp. 529-551, April 1955.

[3] Y.-H. Lee, R.-Y. Kim, and D.-S. Hyun, "A novel SVPWM strategy considering DC-link balancing for a multi-level voltage source inverter," in Proc. I EEE APEC, 1999, vol. 1, pp. 509-514.

[4] G. Venkataramanan and A. Bendre, "Reciprocity transpositionbased sinusoidal pulsewidth modulation for diode-clamped multilevel converters," IEEE Trans. I nd. Electron., vol. 49, no. 5, pp. 1035-1047, Oct. 2002.

[5] E. Sakasegawa and K. Shinohara, "Compensation for neutral point potential in three-level inverter by using motor currents," Trans. Inst.

Eng. Jpn., vol. 121-D, no. 8, pp. 855-861, 2001.

[6] Q. Song, W. Liu, Q. Yu, X. Xie, and Z. Wang, "A neutral point potential balancing algorithm for three-level NPC inverters using analytically injected zero sequence voltage," in Proc. IEEE APEC, Feb. 2003, vol. 1, pp.228-233.

[7] A. Bendre, G. Venkataramanan, V. Srinivasan, and D. Rosene, "Modeling and design of a neutral point voltage regulator for a three level diode clamped inverter using multiple carrier modulation," IEEE Trans. Ind. Electron., vol. 53, no. 3, pp. 718726, Jun. 2006.

[8] A. A. M. Bento, K. V. D. de Almeida, A. R. M. Oliveira, E. R. C. da Silva, and C. B. Jacobina, "A high power factor three-phase three-level rectifier,"in Proc. IEEE PESC, Jun. 17-21, 2007, pp. 3040-3045.

[9] G. Bhuvaneswari and Nagaraju, "Multi-level inverter-A comparative study," IETE J. Res., vol. 51, no. 2, pp. 141-153, Mar./Apr. 2005.

[10] A. Yazdani and R. R. Iravani, “A generalized state space averaged model of the three-level NPC converter for systematic DC voltage balancer and current controller design," IEEE Trans. Power Del., vol. 20, no. 2, pp. 1105-1114, Apr. 2005.

[11] C. Newton and M. Summer, "Neutral point control for multilevel inverters: Theory, design and operational limitations," in Conf. Rec. IEEE IAS Annu. Meeting, New Orleans, LA, USA, Oct. 5-9, 1997, pp. 1336-1343.

[12] C. Newton, M. Summer, and T. Alexander, "Multilevel converters: A real solution to high voltage drives," in IEE Colloq. Update New Power Electron. Tech., May 1997, pp. 3/1-3/5.

[13] H. Akagi and T. Hatada, "Voltage balancing control for a threelevel diode-clamped converter in a medium-voltage transformerless hybrid active filter," IEEE Trans. Power Electron., vol. 24, no. 3, pp.571-579, Mar. 2009

[14] S. Alepuz, J. Bordonau, and J. Peracaula, "Dynamic analysis of three-levelvoltage-source inverters applied to power regulation," in Proc. 30th IEEE PESC, 1999, vol. 2, pp. 721-726.

[15] D. Boroyevich, J. Pou, and R. Pindado, "New feed-forward space vector PWM method to obtain balanced ac voltages in a three level neutral point clamped converter," IEEE Trans. Ind. Electron., vol. 49,no. 5,pp.1026-1034,Oct.2002. 